University of Nebraska - Lincoln

DigitalCommons@University of Nebraska - Lincoln

8-1-1991

\title{
Differential and Total Cross Sections for Ionization of Helium and Hydrogen by Electrons
}

M. Eugene Rudd

University of Nebraska - Lincoln, erudd@unl.edu

Follow this and additional works at: https://digitalcommons.unl.edu/physicsrudd

Part of the Physics Commons

Rudd, M. Eugene, "Differential and Total Cross Sections for lonization of Helium and Hydrogen by Electrons" (1991). M. Eugene Rudd Publications. 37.

https://digitalcommons.unl.edu/physicsrudd/37

This Article is brought to you for free and open access by the Research Papers in Physics and Astronomy at DigitalCommons@University of Nebraska - Lincoln. It has been accepted for inclusion in M. Eugene Rudd Publications by an authorized administrator of DigitalCommons@University of Nebraska - Lincoln. 


\title{
Differential and total cross sections for ionization of helium and hydrogen by electrons
}

\author{
M. E. Rudd \\ Department of Physics and Astronomy, University of Nebraska-Lincoln, Lincoln, Nebraska 68588-0111
}

(Received 25 March 1991)

\begin{abstract}
A comprehensive semiempirical model for singly and doubly differential and total ionization cross sections for electron production by electron impact is presented. The model is discussed in relation to several theoretically required constraints. Available experimental data for hydrogen and helium targets are examined, and recommended values of the data are given for all ranges of primary and secondary energies and angles of ejection.
\end{abstract}

\section{INTRODUCTION}

Integral or total ionization cross section (TCS) data for electron impact on atoms and molecules have accumulated for some 60 years but except for the early experiments of Goodrich [1], data for the same process, differential in the angle and energy of the resulting electrons, did not become available until the work of Opal, Peterson, and Beaty was published in 1971. [2]. Singly differential cross sections (SDCS's) are usually obtained by numerical integration of the doubly differential cross sections (DDCS's) over all angles. The energy distributions of secondary electrons, which are embedded in the SDCS data, are important in studies of steller and upper atmospheric phenomena, plasma fusion work, and in modeling radiation damage in biological tissues and in other materials. Angular distributions of electrons, given by the DDCS data, are of practical interest in studies of radial dose distribution. Differential cross sections for electron ejection are of fundamental interest since they provide a stringent test of theoretical descriptions of ionization.

The total cross-section data have been the subject of several reviews [3-8] and a number of equations have been developed to fit the data [9]. However, there has evidently been only one published work in which the differential and total cross sections were all correlated into a consistent set of data with recommended values given. This is the work of Kim [10] on helium, in which he has selected and modified experimental data to make the data internally consistent as well as being consistent with certain well-known theoretical constraints. The resulting DDCS's were fitted as a function of angle by a series of Legendre polynomials. From his paper, one can obtain the TCS and SDCS's, as well as the DDCS's. The data are presented for selected values of primary energy from 100 to $2000 \mathrm{eV}$. However, the range of secondary energies in this work only extends up to $40 \mathrm{eV}$, while the range of energies of the electrons resulting from an ionizing collision extends from 0 to $T-I$, where $T$ is the primary energy and $I$ is the binding energy of the electron in the target atom or molecule.

Kim's analysis relied on the experimental data available at that time, especially that of Opal et al., [2]. However, since then, new data have become available for helium $[11,12]$, that are in partial disagreement with the earlier experimental data and with Kim's recommended values.

Accurate absolute measurements of the DDCS for electron impact are very difficult to make, as is seen from the fact that existing data, even though carefully taken by competent investigators, disagree in some cases by factors of 2 or more. This lack of agreement, of course, makes it difficult for users of the data. Ab initio theoretical calculations have not yet developed to the point where accurate cross sections can be reliably calculated for a wide range of targets for any given set of parameters. However, the use of carefully constructed semiempirical models offers a useful method for arriving at best values for divergent data. Such models allow averaging, not just for data that happen to be taken at a common set of parameters (in this case, the primary energy, secondary energy, angle, and target), but also permit averaging in a multidimensional space. For example, it will be shown that certain key fitting parameters can be described by universal curves which hold for all primary energies and both targets. By choosing the best fits to such universal curves, data for one target at one primary energy, one angle, and one secondary energy can benefit by being averaged with data for other targets and other values of those parameters.

This paper describes a semiempirical model which gives analytical expressions for the singly and doubly differential and total cross sections which are internally consistent and hold over the entire range of primary and secondary energies up to the relativistic range. The parameters in the model have been determined from the experimental data, taking account of the recent experiments as well as earlier work. While in Kim's analysis he selected a set of data which best fulfilled certain theoretical requirements and then fitted it with series formulas, the present model has the agreement with these requirements built into its structure from the start, and instead of presenting the recommended cross sections only for a selected set of primary and secondary energies, this model gives them in a functional form which holds for any desired set of those parameters. The model should find use among those who need to know the energy and angular distribution of electrons from collisions for modeling 
purposes. While no new physics is presented, those engaged in devising new theoretical descriptions of ionization may find the model useful in understanding the detailed systematics of the process.

Preliminary versions of this model were presented earlier [13-15]. The model has been applied so far only to helium and molecular hydrogen targets in order to avoid the complexities due to inner-shell contributions. It may be possible to extend the model to other targets as well by adding contributions from shells with various binding energies.

\section{SINGLY DIFFERENTIAL CROSS SECTIONS}

When electrons are the incident particles, one must allow for exchange because the scattered primary electrons are indistinguishable from the secondary electrons ejected at the same energy from the target. Some authors introduce an artificial distinction by defining the electrons with energies less than $(T-I) / 2$ to be secondary electrons and designate those with higher energies to be the scattered primary electrons. However, this is an arbitrary and generally unnecessary distinction. In the treatment below no distinction is made and the equations apply equally well to both types of electrons. For convenience, $W$ will be referred to as the energy of the secondary electron but, in fact, will stand for the energy of the detected electron, whether secondary or scattered primary.

Mott [16] has derived an expression for collisions between two free electrons which takes exchange into account. This may be written for a collision with a bound electron [17] as

$$
\begin{array}{r}
\sigma_{M}(W, T)=\frac{4 \pi a_{0}^{2} R^{2}}{T}\left(\frac{1}{(W+I)^{2}}+\frac{1}{(T-W)^{2}}\right. \\
\left.-\frac{1}{(W+I)(T-W)}\right),
\end{array}
$$

where $W$ is the energy of an electron coming from the collisions, $a_{0}$ is the Bohr radius, $I$ is the binding energy, and $R$ is the Rydberg energy. The second term in the equation results from the exchange, while the third comes from interference between the two amplitudes. The Mott cross section for a target with $N$ electrons is

$\sigma_{M}(w, t)=\frac{S}{I t}\left[\frac{1}{(w+1)^{2}}+\frac{1}{(t-w)^{2}}-\frac{1}{(w+1)(t-w)}\right]$,

where $S=4 \pi a_{0}^{2} N(R / I)^{2}$, and where for convenience the equation has been rewritten in terms of the dimensionless variables $w=W / I$ and $t=T / I$. Such variables have been used before under the names "reduced energy" or "threshold units."

Unfortunately, the Mott equation as it stands does not reproduce the experimental SDCS data very closely since its slope at small values of $w$ is too small. Furthermore, the cross sections do not follow the correct $(1 / t) \ln t$ dependence at high energies as derived from the Born approximation by Bethe [18]. This is the dipole interaction term that dominates at high energies.

In the present model, the Mott equation is modified to eliminate these problems, first by changing the powers of the terms in the brackets, and second by replacing the $1 / t$ outside the brackets with a function $F(t)$, which is to be determined by comparison with experiment, and which has the correct asymptotic dependence. The resulting SDCS equation is then

$$
\sigma(w, t)=S F(t) f_{1}(w, t) / I
$$

where

$f_{1}(w, t)=\frac{1}{(w+1)^{n}}+\frac{1}{(t-w)^{n}}-\frac{1}{[(w+1)(t-w)]^{n / 2}}$,

and where $n$ is a number, expected to be somewhat larger than 2 , which is determined by fitting experimental spectra. The use of inverse powers of $W+I$ in describing collision cross sections has been justified theoretically by Inokuti and Dillon [19].

\section{TOTAL IONIZATION CROSS SECTIONS}

Since each collision results in two outgoing electrons, the total ionization cross section is one-half the integral of the SDCS from 0 to the maximum energy $T-I$. Then

$$
\sigma_{\text {ion }}=\frac{1}{2} \int_{0}^{T-I} \sigma(W, T) d W=\frac{I}{2} \int_{0}^{t-1} \sigma(w, t) d w,
$$

where the integrand is given by Eq. (3). The first two terms, which supply most of the integral, are readily integrated, but the third term is not. Of course, numerical integration can always be used, but it is more convenient to have an analytical expression. There is a simple approximation that yields a fairly accurate integral. This involves replacing $t-w$ in the third term by $t-w_{\mathrm{av}}$, where $w_{a v}$ is the average of the maximum and minimum values of $w$, namely $(t-1) / 2$. This approximation for that term alone becomes increasingly inaccurate as the energy is increased, but for high energies that term is too small to contribute appreciably. Setting $t-w=(t+1) / 2$ in that term, the overall integral agrees with the results of numerical integration to within $1 \%$ at all energies for values of $n$ from 1.6 to 2.45 , within $2 \%$ from 1.3 to 3.1 , and is exact at $n=2$. The total cross section with this approximation is

$$
\sigma_{\text {ion }}=S F(t) g_{1}(t),
$$

where

$$
\begin{aligned}
g_{1}(t) & =\frac{1}{2} \int_{0}^{t-1} f_{1}(w, t) d w \\
& =\frac{1-t^{1-n}}{n-1}-\left(\frac{2}{t+1}\right)^{n / 2} \frac{1-t^{1-n / 2}}{n-2} .
\end{aligned}
$$

At $n=2, g_{1}(t)$ becomes indeterminate, but the value of the integral is easily obtained either by integration of the original equation or by finding the limiting value of Eq. 
(7). The result is

$$
g_{1}(t)=\frac{t-1}{t}-\frac{\ln (t)}{t+1} \text { for } n=2 .
$$

While several different functions $F(t)$ may be possible, the one selected is closely related to the Bethe expression [18] and therefore gives cross sections having the proper asymptotic $(1 / t) \ln t$ high-energy dependence. The function is

$$
F(t)=\left(A_{1} \ln t+A_{2}+A_{3} / t\right) / t .
$$

The coefficients $A_{1}, A_{2}$, and $A_{3}$ are taken to be adjustable parameters obtained by fitting to the experimental data. With this choice, the limiting forms of the TCS are

$$
\lim _{t \rightarrow \infty} \sigma_{\text {ion }}=\frac{S A_{1} \ln t}{(n-1) t}
$$

and

$$
\lim _{t \rightarrow 1} \sigma_{\text {ion }}=S\left(A_{2}+A_{3}\right)(t-1) / 2 .
$$

By comparing the high-energy asymptotic behavior of the model equation with the Bethe equation, the parameter $A_{1}$ in the model may be related to the quantity $A_{\text {ion }}$ in the Bethe treatment [18]. This relationship is

$$
A_{\text {ion }}=\frac{N R}{I} \frac{A_{1}}{n-1} \text {. }
$$

The quantity $A_{\text {ion }}$ may be obtained from the integrated optical oscillator strength [18], so $A_{1}$ could also be determined from Eq. (12).

\section{FITTING THE SDCS AND TCS DATA}

The values of $n$, and $F(t)$ could be obtained for each value of primary energy by fitting Eq. (3) to the SDCS as a function of $w$. Spectra at many of the primary energies from most of the known sets of data for helium and molecular hydrogen were fitted to yield data for this study. The equation fits individual runs with an overall average deviation of $8 \%$, which is generally well within the experimental uncertainty. There is, however, a great deal of variation in the values of $F(t)$ among the different investigators, which is indicative of the difficulty in making this type of measurement. The values of $n$ tend to be somewhat more closely grouped and indicate that within the experimental uncertainty, $n$ is a constant, independent of $w$ and $t$, for a given target. The average values of

TABLE I. Values of fitting parameters.

\begin{tabular}{lcc}
\hline \hline & Hydrogen & Helium \\
\hline$A_{1}$ & $0.74 \pm 0.02$ & $0.85 \pm 0.04$ \\
$A_{2}$ & $0.87 \pm 0.05$ & $0.36 \pm 0.09$ \\
$A_{3}$ & $-0.60 \pm 0.05$ & $-0.10 \pm 0.10$ \\
$n$ & $2.4 \pm 0.2$ & $2.4 \pm 0.3$ \\
$G_{5}$ & 0.33 & 0.33 \\
$\beta$ & 0.60 & 0.60 \\
$\gamma$ & 10 & 10 \\
\hline \hline
\end{tabular}

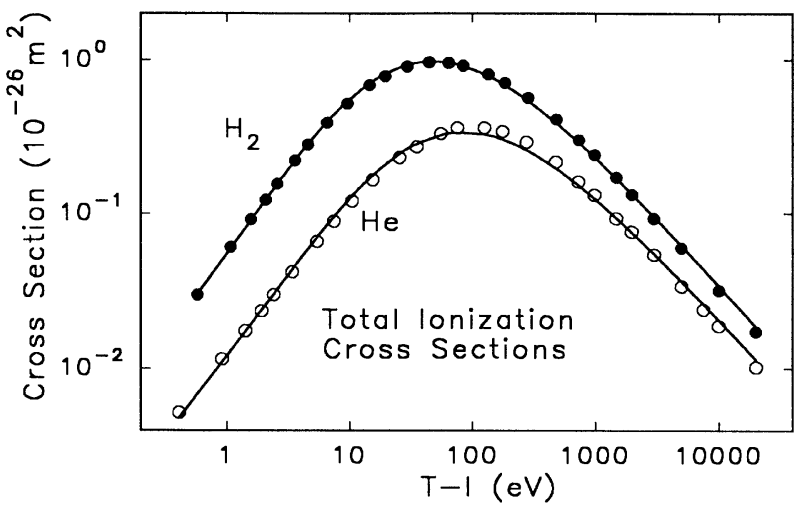

FIG. 1. Total cross sections for $e^{-}+\mathrm{He}$ and $e^{-}+\mathrm{H}_{2}$ vs $T-I$. Lines, Eq. (6); closed circles, hydrogen data; open circles, helium data; experimental values as described in the text.

$n$ determined this way are given in Table I. It is not known whether the fact that $n$ comes out the same for the two gases means that it is a universal constant for all targets or whether this result is simply coincidental.

The coefficients in $F(t)$ were determined from the experimental TCS's, since they have been measured more accurately than the SDCS's. For hydrogen, the data of Rapp and Englander-Golden [20] were used from threshold up to $100 \mathrm{eV}$, the recommended values of van Wingerden et al. [21] from 100 to $2000 \mathrm{eV}$, and the Bethe equation with constants given by van Wingerden et al. [21] for energies above $200 \mathrm{eV}$. For helium, the data of Rapp and Englander-Golden were used below 30 $\mathrm{eV}$, the recommended values of Bell et al. [22] from 30 to $4000 \mathrm{eV}$, and the Bethe equation with constants given by $\mathrm{Kim}$ [23] above $4000 \mathrm{eV}$. Equation (6) was fitted to these data with the resulting values of the parameters as given in Table I. The fitted equation is plotted in Fig. 1 for both targets along with samples of the data mentioned.

\section{DOUBLY DIFFERENTIAL CROSS SECTIONS}

The angular distribution of electrons emitted with a given energy is usually dominated by the binary encounter peak, which is a slice across the so-called Bethe ridge in a three-dimensional plot. It was found that this peak could be represented by a Lorentzian in the cosine of the angle. An additional Lorentzian centered at $180^{\circ}$ was used to fit the rise in the cross section often noted at large angles. The equation is

$$
\sigma(w, t, \theta)=G_{1}\left[f_{\mathrm{BE}}(w, t, \theta)+G_{4} f_{b}(w, t, \theta)\right],
$$

where

$$
f_{\mathrm{BE}}(w, t, \theta)=\frac{1}{1+\left[\left(\cos \theta-G_{2}\right) / G_{3}\right]^{2}}
$$

and

$$
f_{b}(w, t, \theta)=\frac{1}{1+\left[(\cos \theta+1) / G_{5}\right]^{2}},
$$


and where $G_{1} \ldots G_{5}$ are the fitting parameters, which, in general, are functions of the primary and secondary energies.

There is some question as to whether the rise seen in the backward direction is physical or instrumental. While the last term in Eq. (13) is needed to fit some of the data above about $120^{\circ}$, there are large variations among the data of different investigators. If it is later decided that this rise is not real, the model can easily be modified by omitting or reducing the size of that term.

From the data available, angular distributions for over 100 different combinations of primary and secondary energy, target gas, and investigator were selected and fitted by Eq. (13) to obtain information on the systematics of the parameters.

The parameter $G_{2}$ is given by $G_{2}=\cos \theta_{0}$, where $\theta_{0}$ is the position of the center of the binary peak. This is determined by the laws of conservation of energy and momentum applied to a collision of an incident electron with a bound, stationary electron. If the collision is assumed to be perfectly elastic, but the electron subsequently loses the energy $I$ as it leaves the atom or molecule, then the equation given by $\mathrm{Kim}$ [10] results in

$$
G_{2}=\left(\frac{w+1}{t}\right)^{1 / 2}
$$

The results of fitting the experimental data are shown for the parameter $G_{2}$ in Fig. 2, where they are compared to the predictions of Eq. (16). Since the binary peak is very broad at small secondary energies, it is difficult to find the center, hence the large spread in values of $G_{2}$ at low energies.

The width of the peak results from the distribution in

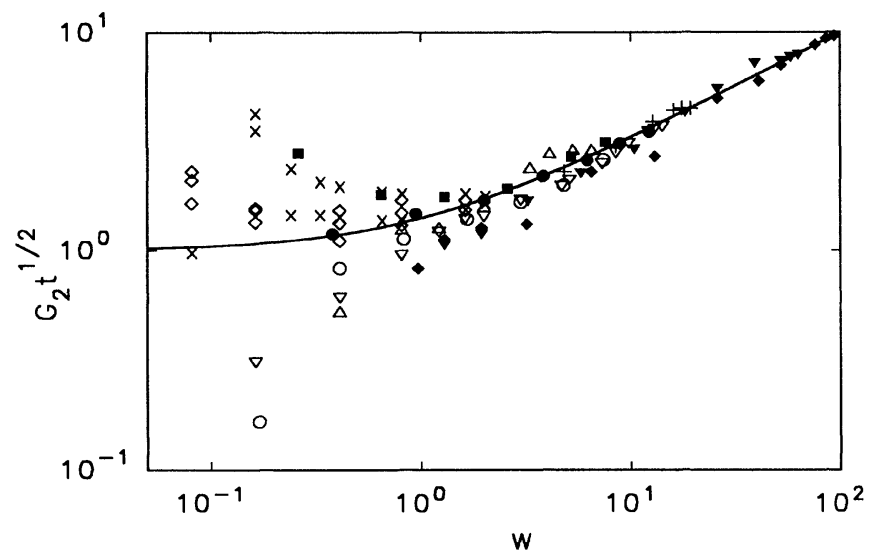

FIG. 2. Values of the parameter $G_{2}$, plotted as $G_{2} t^{1 / 2}$ vs $w$, obtained by fitting to the experimental data. Solid line, Eq. (16); closed symbols, hydrogen, as follows: squares, Shyn, Sharp, and Kim [24]; circles, Opal, Peterson, and Beaty [2]; inverted triangles, Hollman et al. [25]; diamonds, Rudd, Lewis, and Kerby [14]. Open symbols, helium, as follows: diamonds, MuellerFiedler, Jung and Erhardt [12]; $x$ 's, Goruganthu and Bonham [11]; inverted triangles, Sethuraman, Rees, and Gibson [26]; crosses, Oda [27]; triangles, Rudd and DuBois [28]; circles, Opal, Peterson, and Beaty [2]. direction and velocity of the initial motion of the orbital electrons in the target. The shape of the binary peak has, in fact, been used to determine the Compton profile $[29,30]$, which is the distribution of momentum in the target. $G_{3}$, the half width at half maximum of the cosine distribution, may be written

$$
G_{3}=\Delta \cos \theta \simeq \sin \theta_{0} \Delta \theta
$$

The value of $\Delta \theta$ may be estimated by assuming the initial velocity of the orbital electrons to be $(2 I / \mathrm{m})^{1 / 2}$ and that this velocity is added vectorially at right angles to the velocity $(2 \mathrm{~W} / \mathrm{m})^{1 / 2}$ of the ejected electrons. To be more accurate, of course, the orbital kinetic energy $U$ should be used, since it is usually significantly different from the ionization potential $I$. However, values of $U$ are not measurable quantities and must be calculated. Since such calculations are not readily available and only a functional dependence is needed at this point, $I$ is used instead of $U$. If $I \ll W$, then $\Delta \theta=(I / W)^{1 / 2}=(w)^{-1 / 2}$. However, this neglects the fact that there is a distribution both in direction and magnitude of the orbital velocities, and therefore the actual half-width should be smaller by some factor. Combining these considerations, then,

$$
G_{3}=\beta\left(\frac{1-G_{2}^{2}}{w}\right)^{1 / 2} .
$$

By comparison with experiment, $\beta=0.60$.

The values of $G_{3}$ from the fitting are shown in Fig. 3 compared with the expectations from Eq. (18). Although the equation was derived under the assumption that the orbital velocity is small compared to the ejection velocity, the equation appears to fit both the hydrogen and helium data quite well to as low an energy as the reliable experimental data go. Furthermore, as scaled, the equation appears to be a universal equation holding for all primary energies and for both gases.

The parameters $G_{4}$ and $G_{5}$ are determined from fitting the angular distributions above $90^{\circ}$. Unfortunately, the discrepancies among the experimental data sets are even more pronounced at the large angles than elsewhere and

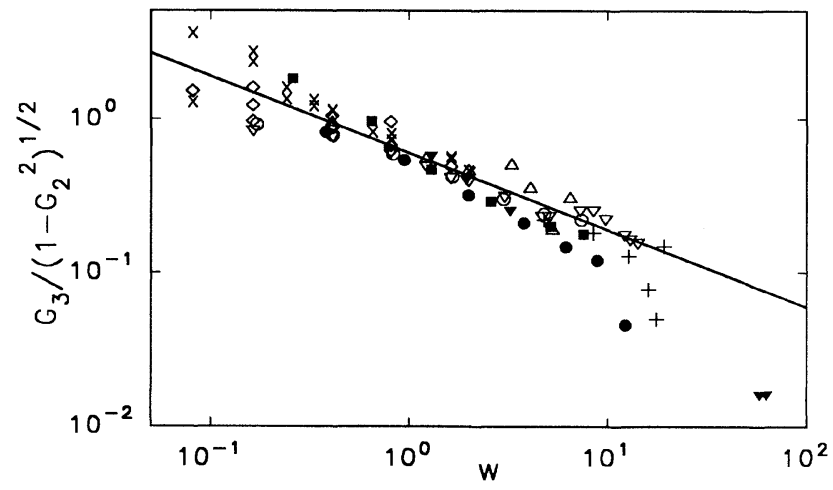

FIG. 3. Values of the parameter $G_{3}$ obtained by fitting to the experimental data, plotted as $G_{3} /\left(1-G_{2}^{2}\right)^{1 / 2}$ vs $w$. Line, Eq. (18); symbols as in Fig. 2. 
in some cases data are not presented at angles close enough to $180^{\circ}$ to obtain reliable values of these parameters. Consequently, it is difficult to find the systematics of $G_{4}$ and $G_{5}$. Fortunately, except for the lower impact energies, the rise at large angles is only a minor part of the overall angular distribution. A rough approximation will have to suffice for this region until more and better data are available.

Many of the data sets show only a small rise in the backward direction or none at all. However, the latest data, that of Müller-Fiedler [12] and of Gorguganthu and Bonham [11], have a pronounced rise near $180^{\circ}$. Data for low secondary energies from these investigators and data at higher energies from Oda [27] were fitted by Eq. (13) to obtain values of $G_{5}$ with the results shown in Fig. 4. Even with this restricted sample, $G_{5}$ exhibits large variations. Although it appears that lower values of $G_{5}$ would be appropriate at higher energies, there is not enough data to assign values with confidence. Instead, we will use the constant value $G_{5}=0.33$, which represents a weighted average of the data shown.

Using the fixed value of $G_{5}$, the data were refitted to obtain information about $G_{4}$. That parameter decreases with increasing $w$, slowly at first, and then drops rapidly as $w \rightarrow t-1$. The variation of $G_{4}$ with $w$ and $t$ is approximated by the expression

$$
G_{4}=\gamma \frac{(1-w / t)^{3}}{t(w+1)}
$$

The quantity $\gamma$ is a constant chosen for the best fit of the data. For the hydrogen and helium data, $\gamma=10$ was used. Figure 5 shows the values of $G_{4}$ from the fitting along with the line that represents Eq. (19). Although the general trend of the data follows the line, there are large variations among the data. Indeed, the data of Opal et al. [2] have a rising rather than a falling characteristic in the plot.

The parameter $G_{1}$ will be discussed in the next section.

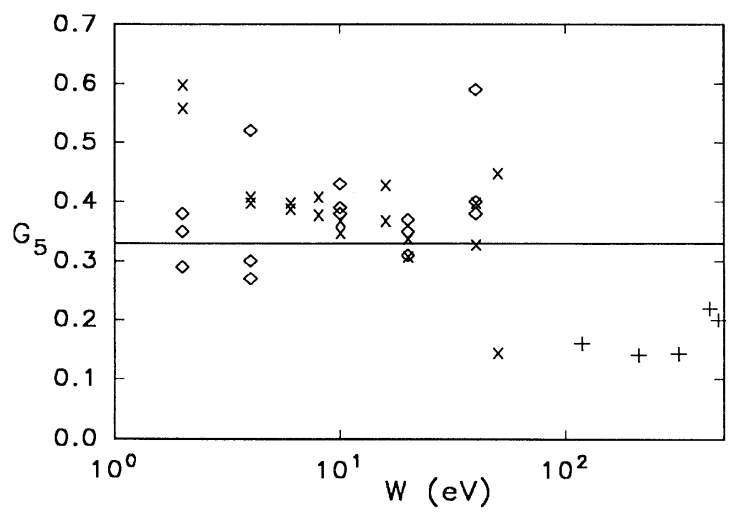

FIG. 4. Values of the parameter $G_{5}$ obtained by fitting to the experimental data, plotted vs $W$. The line represents the weighted average, 0.33, used in the model; symbols as in Fig. 2.

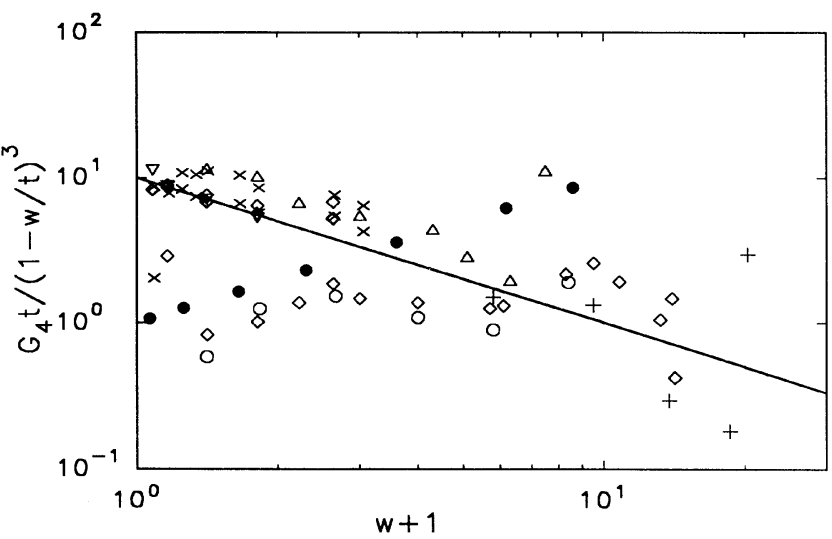

FIG. 5. Values of the parameter $G_{4}$ obtained by fitting to the experimental data, plotted as $G_{4} t /(1-w / t)^{3}$ vs $w+1$. Line, Eq. (19); symbols as in Fig. 2.

\section{INTEGRATION OF THE DDCS EQUATION}

The DDCS may be integrated over angle to obtain the SDCS. Thus

$$
\sigma(w, t)=\int \sigma(w, t, \theta) d \Omega,
$$

where $\sigma(w, t, \theta)$ is given by Eq. (13). The result is

$$
\sigma(w, t)=G_{1}\left[g_{\mathrm{BE}}(w, t)+G_{4} g_{b}(w, t)\right],
$$

where

$$
\begin{aligned}
g_{\mathrm{BE}}(w, t)= & \int f_{\mathrm{BE}}(w, t, \theta) d \Omega \\
=2 \pi G_{3} & {\left[\tan ^{-1}\left(\frac{1-G_{2}}{G_{3}}\right)\right.} \\
& \left.+\tan ^{-1}\left(\frac{1+G_{2}}{G_{3}}\right)\right]
\end{aligned}
$$

and

$$
g_{b}(w, t)=\int f_{b}(w, t, \theta) d \Omega=2 \pi G_{5} \tan ^{-1}\left(\frac{2}{G_{5}}\right) .
$$

For $G_{5}=0.33, g_{b}=2.9$, a constant.

The integral of the DDCS given in Eq. (21) may be equated to the expression for the SDCS given in Eq. (3) to obtain $G_{1}$ :

$$
G_{1}=\frac{S F(t) f_{1}(w, t) / I}{g_{\mathrm{BE}}(w, t)+G_{4} g_{b}(w, t)} .
$$

Values of $G_{1}$ obtained by fitting Eq. (13) to the data sets for helium at $500 \mathrm{eV}$ are shown in Fig. 6 compared to the calculations from Eq. (24).

\section{REQUIREMENTS ON THE MODEL}

Kim [10] has given six major requirements that must be satisfied by a consistent set of DDCS data. We next 


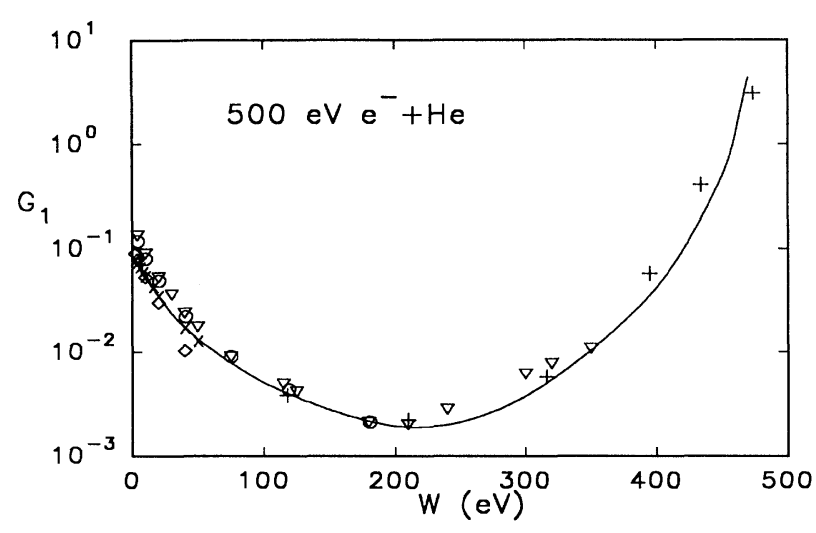

FIG. 6. Values of the parameter $G_{1}$ obtained by fitting to the 500-eV experimental data for helium, plotted vs $W$. Line, Eq. (24); symbols as in Fig. 2.

examine the present model to see how well it satisfies those requirements.

\section{A. Threshold behavior}

Clearly, all of the cross sections must go to zero at threshold, i.e., at $t=1$. In the present model, $g_{1}(t)$ goes to zero at $t=1$, thus insuring that the cross sections obey this requirement. Furthermore, as $t \rightarrow 1$ the TCS expression calculated from the model has a linear dependence on the excess energy above threshold, thus agreeing quite well with the 1.127 power dependence derived by Wannier [31].

\section{B. Asymptotic behavior}

As already mentioned, through the high-energy behavior of $F(t)$, the TCS expression has the required $(1 / t) \ln t$ dependence. The SDCS and DDCS have the same asymptotic dependence.

Kim also noted [10] that the Bethe approximation yields a $\sin ^{2} \theta$ angular dependence at high energies. If $t \gg 1$ and $w<<t$, then $G_{4}<<1$, and the first term of Eq. (13) dominates. Under these conditions, $G_{2} \ll 1$ and $G_{3}>1$. Then, from Eq. (14),

$$
f_{\mathrm{BE}}(w, t, \theta) \simeq \frac{1}{1+\cos ^{2} \theta / G_{3}^{2}} .
$$

Using the first two terms of the binomial expansion,

$f_{\mathrm{BE}}(w, t, \theta) \simeq 1-\frac{\cos ^{2} \theta}{G_{3}^{2}}=$ const + const $\times \sin ^{2} \theta$,

in agreement with the Bethe requirement.

\section{Angular symmetry}

As just shown, the model yields a $\sin ^{2}$ angular dependence at high energies, thus automatically satisfying the requirement of back-front symmetry in that limit.

\section{Integrated cross sections}

This constraint requires the integral of the differential cross section to agree with the TCS and for the latter to have the proper $(1 / t) \ln t$ high-energy dependence. As noted, these properties are built into the model in the function $F(t)$. Furthermore, the value of $A_{1}$, as described earlier, is related to the integrated optical oscillator strength.

\section{E. Energy-loss cross section}

The first moment of the energy distribution is closely related to the stopping cross section. This is defined as

$$
\sigma_{\mathrm{st}}(T)=\frac{1}{R} \int_{0}^{(T-I) / 2}(W+I) \sigma(W, T) d W .
$$

The asymptotic high-energy limit of this integral obtained from the model should agree with the prediction of the Bethe theory. For high energies, only the first terms of $f_{1}(w, t)$ and of $F(t)$ are needed. Doing the integral and dropping higher-order terms in $1 / t$, the model yields

$$
\sigma_{\mathrm{st}}=\frac{4 \pi a_{0}^{2} R N A_{1} \ln T}{(n-2) T} .
$$

The leading term in the Bethe equation is [18]

$$
\sigma_{\mathrm{st}}=\frac{8 \pi a_{0}^{2} N R \ln T}{T} .
$$

Comparing the two equations, $A_{1} /(n-2)=2$. Using the values of $A_{1}$ from Table I, $A_{1} /(n-2)=2.1$ and 1.9 for helium and hydrogen, respectively, both in good agreement with the expected value.

\section{F. Binary peak}

Since the target electron is bound, the equation for the center of the binary peak is not given by $\cos \theta_{0}=(w / t)^{1 / 2}$, but rather by Eq. (16), as indicated previously. This dependence is part of the model.

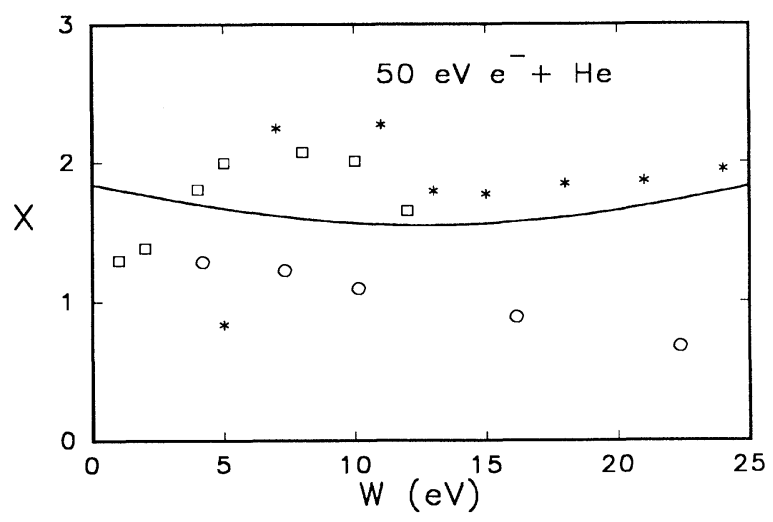

FIG. 7. SDCS for $50-\mathrm{eV} e^{-}+\mathrm{He}$ collisions plotted as $X$ (see text) vs $W$. Line, Eq. (3); asterisks, Crooks [32]; other symbols as in Fig. 2. 


\section{RECOMMENDED CROSS SECTIONS}

The parameter $n$ for the two gases was determined by fitting to the SDCS, while $A_{1}, A_{2}$, and $A_{3}$ were found by fitting the TCS. The values of the parameters are given in Table I. The TCS may then be calculated for any primary energy by Eq. (6). Using the same parameters, Eq. (3) gives the SDCS recommended for the two gases for any combination of primary and secondary energies. In Figs. 7-9 the results of the model are compared with experimental values of the SDCS. To reduce the large variation of the SDCS with energy, the measured and calculated cross sections have been divided by the Mott equation values given by Eq. (1). Thus, the quantity $X=\sigma(W, T) / \sigma_{M}(W, T)$ is plotted. This is similar to the quantity $Y$, the ratio of the SDCS to the Rutherford cross section, often used, but has the advantage of being symmetric about $(T-I) / 2$.

At low primary energies, complete collection of the primary beam is very difficult because of scattering and space-charge spreading. Furthermore, the resulting secondary electrons, which are necessarily also of low energies, are hard to analyze and detect with uniform efficiency, and spurious electrons are more numerous at those energies. For these reasons, it is not surprising that there is a large spread of values among the 50-eV data points of various investigators, as seen in Fig. 7. The present model provides a way of extrapolating the more reliable data at higher energies to the low-energy region and therefore may in some cases be more accurate than the experimental data.

Figures 8 and 9 show that for the helium SDCS data at 200 and $2000 \mathrm{eV}$, there is very good agreement between the model and the recommended values of Kim [10] indicated by the dotted line. There is some discrepancy between the model and the data of Opal, Peterson, and Beaty [2] for $W>50 \mathrm{eV}$ in the $2000-\mathrm{eV}$ results. Since there are no other experimental data in that region, it is not possible to say whether the error is in the model or in the data. However, data from that group tend to be too low in the intermediate range of electron energies com-

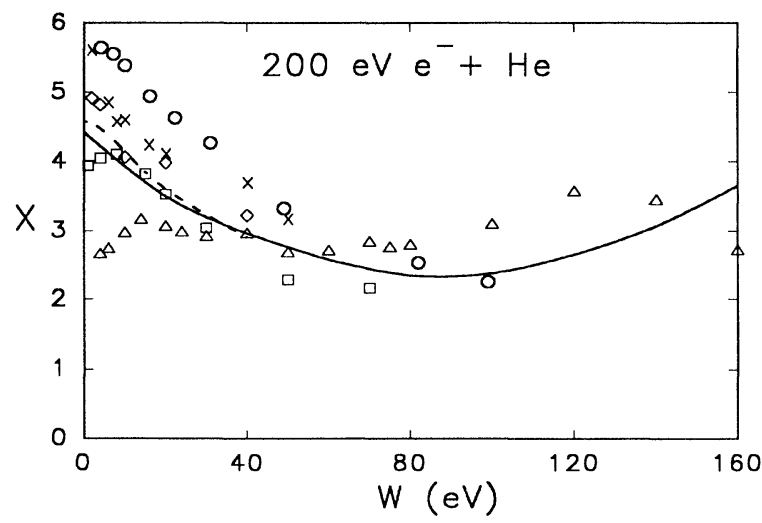

FIG. 8. SDCS for $200-\mathrm{eV} e^{-}+\mathrm{He}$ collisions plotted as $X$ vs $W$. Dashed line, Kim [10]; solid line, Eq. (3); symbols as in Fig. 2 .

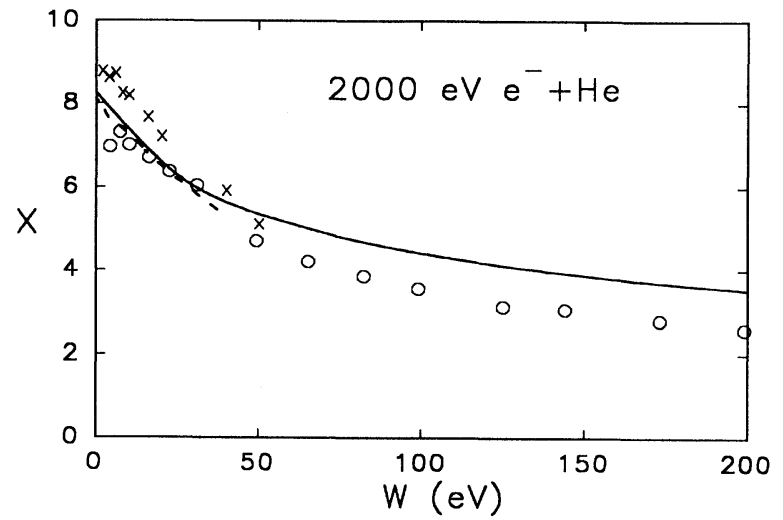

FIG. 9. SDCS for 2000-eV $e^{-}+\mathrm{He}$ collisions plotted as $X$ vs $W$. Dashed line, Kim [10]; solid line, Eq. (3); symbols as in Fig. 2.

pared to other experimental data as seen, e.g., in Figs. 7 and 8.

For the DDCS, the parameter $G_{1}$ is obtained from Eq. (24), $G_{2}$ from Eq. (16), $G_{3}$ from Eq. (18), and $G_{4}$ from Eq. (19). $G_{5}$ has the fixed value 0.33 . Then the DDCS for any combination of primary and secondary energy and angle is given by Eq. (13). Examples of the comparison of the model with experiment and with Kim's recommended values [10] are given in Figs. 10-12. In most cases, the model agrees reasonably well with the data when the relatively large spread among the experimental values is taken into account. The discrepancies are somewhat greater at low primary energies. Since two investigators have found that a peak in their data at zero angle was due only to spurious electrons $[25,35]$, the rise in the cross section seen in the forward direction in some of the experimental data (not shown here) is probably not real. The agree-

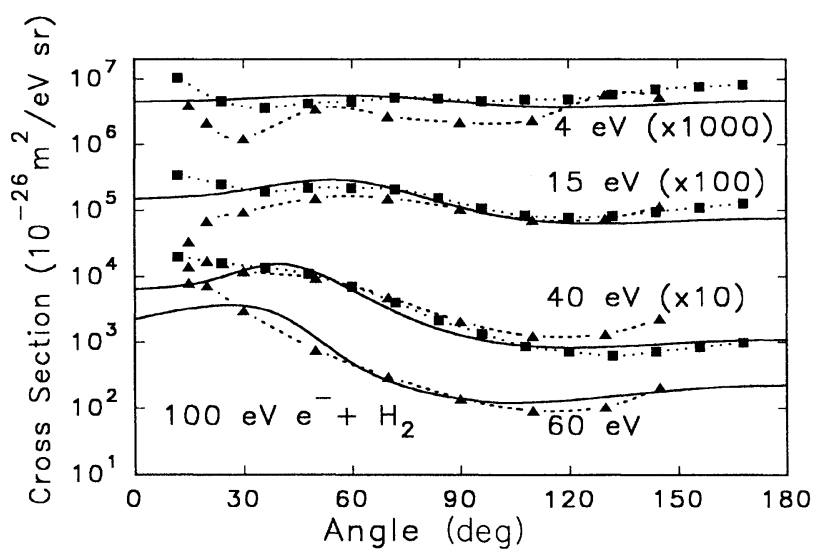

FIG. 10. DDCS for $100-\mathrm{eV} e^{-}+\mathbf{H}_{2}$ collisions plotted vs angle $\theta$ for various ejection energies. Line, Eq. (13); closed squares, Shyn and Sharp [33]; closed triangles, DuBois and Rudd [34]. Dotted lines are only to guide the eye. 


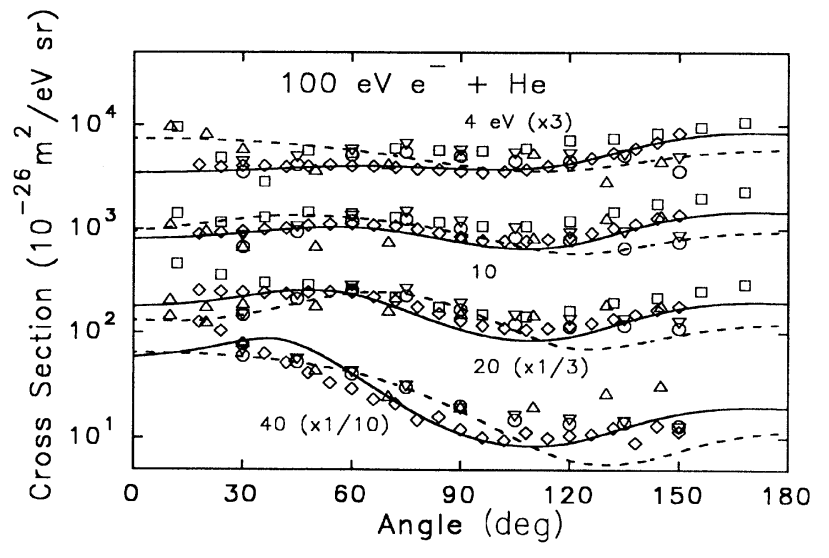

FIG. 11. DDCS for $100-\mathrm{eV} e^{-}+\mathrm{He}$ collisions plotted vs angle $\theta$ for various ejection energies. Solid line, Eq. (13); dashed line, Kim [10]; symbols as in Fig. 2.

ment with Kim's recommended values [10], both SDCS and DDCS, is very good for the higher primary energies, but the agreement between the two angular distributions is not as good at lower energies. From Fig. 11 it appears that for lower energies the present model follows the shape of the data of Müller-Fiedler, Jung, and Erhardt [12] better than Kim's values do.

\section{CONCLUSIONS}

A model has been presented by means of which cross sections for electron-impact ionization, either total or differential in energy and angle of ejection, are given in compact form for all combinations of primary and secondary energy and angle. Parameters have been obtained for helium and hydrogen targets from fitting to experimental data. While the model seems to be successful for those targets, it should also be tested for heavier targets for which there is a more complex energy dependence of the dipole oscillator strengths. While the dipole term is important at intermediate and high primary energies, it is of lesser importance at low energies. Therefore, the mod-

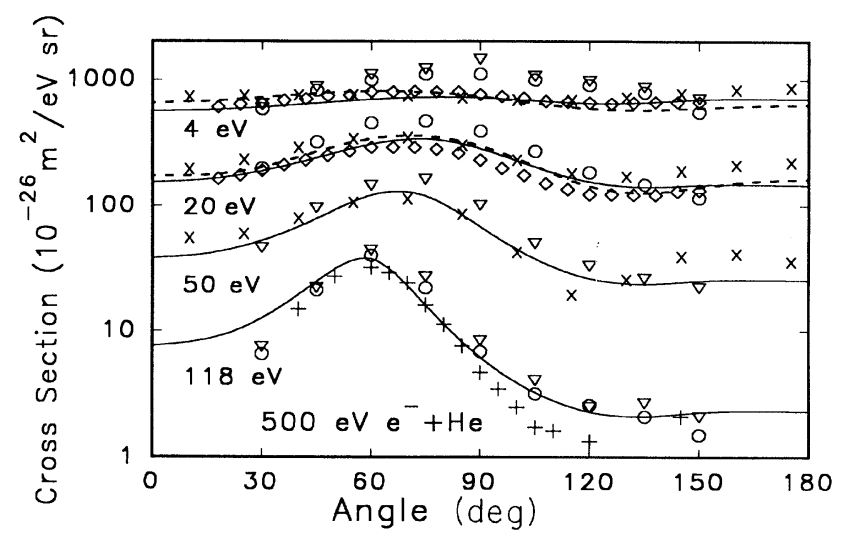

FIG. 12. DDCS for $500-\mathrm{eV} e^{-}+\mathrm{He}$ collisions plotted vs angle $\theta$ for various ejection energies. Solid line, Eq. (13); dashed line, Kim [10]; symbols as in Fig. 2.

el may be especially useful at low energies where the high-energy models, such as those based on the Bethe equation, fail.

This analysis points up the need for additional experimental data and also the lack of complete theoretical understanding of the electron emission process. Questions concerning the nature of systematics of the rise in the backward direction, the dependence of the shape of the binary encounter peak on primary and secondary energies, and the exact functional dependence of the SDCS need to be addressed.

\section{ACKNOWLEDGMENTS}

The author is pleased to acknowledge helpful discussions and a key suggestion by Keith Long concerning the singly differential cross-section model. He also thanks Mitio Inokuti, Larry Toburen, Russell Bonham, and John Miller for their helpful comments and suggestions. Continuing discussions with Yong-Ki Kim have been very valuable. This work was supported by the National Science Foundation under Grant No. PHY-8701905.
[1] Max Goodrich, Phys. Rev. 52, 259 (1937).

[2] C. B. Opal, W. K. Peterson, and E. C. Beaty, J. Chem. Phys. 55, 4100 (1971). See also, C. B. Opal, E. C. Beaty, and W. K. Peterson, At. Data Nucl. Data Tables 4, 209 (1972).

[3] F. J. de Heer and M. Inokuti, in Electron Impact Ionization, edited by T. D. Märk and G. H. Dunn (SpringerVerlag, Berlin, 1985), p. 232.

[4] H. Tawara and T. Kato, At. Data Nucl. Data Tables 36, 167 (1987).

[5] Isao Shimamura, Scientific Papers Inst. Phys. Chem. Res. 82, 1 (1989).

[6] T. D. Märk, in Electron-Molecule Interactions and Their Applications, edited by L. G. Christophorou (Academic,
Orlando, 1984), Vol. I, Chap. 3, p. 251.

[7] Sandor Trajmar and David F. Register, in Electron Molecule Collisions, edited by K. Takayanagi and I. Shimamura (Plenum, New York, 1983).

[8] L. J. Kieffer and Gordon H. Dunn, Rev. Mod. Phys. 38, 1 (1966).

[9] See, e.g., Wolfgang Lotz, Z. Phys. 206, 205 (1967); 216, 241 (1968).

[10] Yong-Ki Kim, Phys. Rev. A 28, 656 (1983).

[11] R. R. Goruganthu and R. A. Bonham, Phys. Rev. A 34, 103 (1986).

[12] R. Müller-Fiedler, K. Jung, and H. Erhardt, J. Phys. B 19, 1211 (1986)

[13] M. E. Rudd, Nucl. Tracks Radiat. Meas. 16, 213 (1989). 
[14] M. E. Rudd, J. K. Lewis, and G. W. Kerby III, Bull. Am. Phys. Soc. 35, 1147 (1990).

[15] M. E. Rudd, Nucl. Instrum. Methods B 56/57, 162 (1991).

[16] N. F. Mott, Proc. R. Soc. London 126, 259 (1930). See also, L. D. Landau and E. M. Lifshitz, Quantum Mechanics: Non-Relativistic Theory, 2nd ed. (Pergamon, London, 1965), p. 305.

[17] Yong-Ki Kim, Rad. Res. 61, 21 (1975).

[18] H. A. Bethe, Ann. Phys. (Leipzig) 5, 325 (1930). See also, M. Inokuti, Rev. Mod. Phys. 43, 297 (1971); 50, 23 (1978).

[19] Mitio Inokuti and Michael A. Dillon, J. Chem. Phys. 87, 6967 (1987).

[20] D. Rapp and P. Englander-Golden, J. Chem. Phys. 43, 1464 (1965).

[21] B. van Wingerden, R. W. Wagenaar, and F. J. de Heer, J. Phys. B 13, 3481 (1980).

[22] K. L. Bell, H. B. Gilbody, J. G. Hughes, A. E. Kingston, and F. J. Smith, J. Phys. Chem. Ref. Data 12, 891 (1983).

[23] Y.-K. Kim, in Physics of Ion-Ion and Electron-Ion Collisions, edited by F. Brouillard and J. W. McGowan (Plenum, New York, 1983), p. 101.

[24] T. W. Shyn, W. E. Sharp, and Y.-K. Kim, Phys. Rev. A
24, 79 (1981).

[25] K. W. Hollman, G. W. Kerby III, M. E. Rudd, J. H. Miller, and S. T. Manson, Phys. Rev. A 38, 3299 (1988).

[26] S. K. Sethuraman, J. A. Rees, and J. R. Gibson (unpublished).

[27] Nobou Oda, Rad. Res. 64, 80 (1975); (private communication).

[28] M. E. Rudd and R. D. DuBois, Phys. Rev. A 16, 26 (1977).

[29] H. Bökl and F. Bell, Phys. Rev. A 28, 3207 (1983).

[30] A. D. Barlas, W. H. E. Rueckner, and H. F. Wellenstein, J. Phys. B 11, 3381 (1978).

[31] Gregory H. Wannier, Phys. Rev. 90, 817 (1953).

[32] G. B. Crooks, Ph.D. thesis, University of Nebraska, 1972 (unpublished)

[33] T. W. Shyn and W. E. Sharp, Phys. Rev. A 19, 557 (1979).

[34] R. D. DuBois and M. E. Rudd, Phys. Rev. A 17, 843 (1978).

[35] N. Oda and F. Nishimura, in Abstracts of the Tenth International Conference on the Physics of Electronic and Atomic Collisions, edited by M. Barat and J. Reinhardt (Commissariat a L'Energie Atomique, Paris, 1977), p. 362. 